\title{
Evaluation of pharmaceutical intervention in patients with hepatic insufficiency in a hospital setting
}

\begin{abstract}
Background: Dose adjustment in patients with hepatic insufficiency (HI) is not routinely performed in clinical practice by the difficult to quantify the liver dysfunction and the lack of dosage data for many drugs in patients with HI. There is scarce evidence on support prescription tools to identify these patients and make recommendations about their medication management.
\end{abstract}

Objective: To assess the prevalence of potentially inappropriate prescriptions susceptible to a pharmacist intervention (PI) and evaluate their satisfaction with the intervention based on the Child-Pugh (CP) and perceptions of its impact.

Setting: A Spanish large, tertiary care and academic medical hospital.

Method: This uncontrolled prospective evaluation of pharmaceutical intervention was screened patients with $\mathrm{HI}$ according to the calculation of the analytical blood test variables score of the Child-Pugh score (ACP) $>7$. The pharmacist reviewed all the order prescriptions considered potentially inappropriate to the clinical situation of liver failure. PI was classified based on five categories according to the recommendation and recorded in order to evaluate the efficiency, the acceptance rate by the clinicians and the clinical impact of negative outcomes related to medication.

Main outcome measure: Measure prevalence of potentially inappropriate prescriptions in patients with $\mathrm{HI}$ and categories according the PI done.

Results: A total of 8815 patients were screened over a period of 6 months, 171 of them had an ACP value $>7 ; 141$ patients have exclusion criteria and our potential interventional population was 30 patients. The HI prevalence rate obtained by the CPT screening was $0.34 \%$ of the recorded hospital admissions and 318 order prescriptions, were candidates for a PI. Once clinical variables (ascites/encephalopathy) and the clinical history had been reviewed the final CP classification was: $\mathrm{B}(11$ patients) and $\mathrm{C}(19$ patients).Regarding the impaired liver function, $58.5 \%$ of orders were considered for potential PI, associated with a higher probability of drug related problem: dosage/frequency change: 67 orders, pharmacological/analytical monitor:47, caution:43 and contraindications:29. On average we recorded a 0.97 (29/30) contraindicated
Volume 9 Issue 6 - 2018

\author{
Leonor Periáñez Párraga,' Pere Ventayol \\ Bosch,' Iciar Martínez-López,' Olga Delgado \\ Sánchez,' Jesus Martínez Sotelo, ${ }^{2}$ Miguel \\ Ángel Calleja Hernández ${ }^{3}$ \\ 'Pharmacy Service, Hospital Universitari Son Espases, Palma de \\ Mallorca-Illes Balears, Spain \\ ${ }^{2}$ Pharmacy Service, Hospital Comarcal d'Inca, Inca-Illes Balears, \\ Spain \\ ${ }^{3}$ Pharmacy Service, Hospital Universitario Virgen de las Nieves, \\ Spain
}

Correspondence: Leonor Periañez Parraga, Carretera Valldemossa $n^{\circ} 79,07$ I 20 Palma, Spain, Tel (+34) 87I.20.54.09, Fax (+34) 871.20.52.50,Email leonard.perianez@ssib.es

Received: July 30, 2018 | Published: November 20, 2018

order/patient. PI acceptance was $69.7 \%$. The PI performed had a potential impact on negative outcomes related to medication; drug efficacy and safety on $12.6 \%$ and $87.4 \%$ respectively.

Conclusion: The results of our study show that about one third of the prescriptions in patients with $\mathrm{HI}$ are candidates for PI, mostly due to contraindications or wrong dosage. As the pharmacists' intervention is widely accepted and appreciated by the medical community they have an important role to play by increasing the safe use of drugs.

Keywords: hospital, medication safety, hepatic insufficiency, inappropriate prescriptions, spain

\section{Impact of findings on practice}

i. There is a lack on drug safety in patients with HI.

ii. ACP is an effective and reproducible pre-screening score to assess the prevalence of prescriptions susceptible to be adjusted in patients with HI.

iii. The pharmacist plays an important role implementing programs to stratify patients and recommending appropriate dose in HI.

\section{Introduction}

The inclusion of liver function parameters in routine clinical prescription has been conditioned by the difficulty to quantify the liver dysfunction and the lack of dosage data for many drugs in patients with Hepatic Insufficiency (HI).

Liver disease represents a clinical setting in terms of drug therapy because it encompasses a range of pathophysiological changes that place patients with this condition at greater risk of adverse reactions to drugs. Indeed, hepatic insufficiency has a significant impact on the pharmacokinetic behavior of many drugs, mainly through reduction in clearance processes, and thus may lead to drug accumulation and related undesired effects, provided that the latter are dosagedependent. ${ }^{1} \mathrm{HI}$ is also one of the disease states that alter the normal relationship between serum drug concentration and response. A general principle is that the pharmacological response to a drug is a function of its free fraction of some drugs, as a result of a reduced serum albumin concentration, is one of the well-known effects of cirrhosis. Additionally, alterations in end-organ responsiveness are encountered in patients with $\mathrm{HI}$ and account for their increased sensitivity to some drugs. HI involves a number of pathophysiological changes, including parenchymal damage, altered hepatic blood flow (mainly caused by the development of portosystemic vascular shunts) and reduced drug binding in blood. All of these factors influence distribution and clearance of drugs and their free concentrations in plasma, although they may be affected to a different extent in patients with the same liver disease process. ${ }^{1}$

Drugs are metabolized and excreted almost exclusively by the liver and the kidneys. Failure of one of these organ systems may have an important impact on patient safety and drug dose adjustment. In patients with chronic renal insufficiency, it has been well established that dosages of nephrotoxic and renally excreted drugs should be adjusted, and some drugs should probably be avoided altogether. ${ }^{2,3}$ In 
these patients, rates of adverse drug events have recently been shown to reach $10 \%$ of admissions. ${ }^{4}$ Many drugs are hepatically metabolized, and it stands to reason that it might make sense to alter dosages and avoid certain medications in patients with CLD, although it has been much less clear how important this is relative to the situation in chronic renal insufficiency.

Pharmaceutical Intervention (PI) is the process of identifying a drug related problem (DRP) and making a recommendation in an attempt to prevent or resolve it. Pharmacotherapy monitoring is the practice in which the pharmacist is responsible for the patient's needs related to medications, which is usually made by detecting DRP preventing and solving negative outcomes related to medication (NOM). Patients with HI should be considered for pharmaceutical monitoring. As a general rule drug recommendations with hepatic metabolism or an elimination of more than $20 \%{ }^{5}$ should be considered for pharmaceutical monitoring. Finally those drugs with a narrow therapeutic range especially in patients with elevated Child-Pugh score $(\mathrm{CP})^{5,6}$ may be considered.

Published data on the administration of medications in patients with $\mathrm{HI}$ in the clinical setting are rare. Approximately $40 \%$ of the medications prescribed during the hospital stay should be used cautiously in patients with HI, which was more prevalent than medications deemed safe in HI. Recently study ${ }^{7}$ shows about $6 \%$ of the administered medications during hospitalization were contraindicated in $\mathrm{HI}$, and increased to $9 \%$ on discharge. In addition $19 \%$ of the medications prescribed during the hospital stay and $27 \%$ of medications on discharge were contraindicated in severe hepatic impairment, according to the official product information.

The CP score was developed in the 70 s to predict mortality rates of emergency portacaval shunt for the treatment of esophageal variceal gastrointestinal bleeding ${ }^{8}$ and, has been the most widely used prognostic index, despite its limitations: not all the measures have an independent effect, some variables are subjective (ascites and encephalopathy), the cut-off points of the quantitative variables are not optimal and does not take into account the renal function and certain important prognostic factors.

Unfortunately, there are no sensitive and specific clinical or biochemical criteria that determine the severity of liver function impairment. Although the biochemical markers are useful indicators of liver disease processes such as active hepatocellular injury or synthetic function, they are not reliable measures of specific metabolic functions such as biotransformation. Indices of hepatic synthetic function are dependent on other variables that can be abnormal in patients with liver disease as well as in other disease states. Although relationships between the clearance of some drugs and the latter indexes of liver dysfunction have occasionally been recorded, these findings have turned out to inconsistent and not reproducible.

However the European Medicine Agency (EMA) and the Food and Drug Administration (FDA) have by consensus established that the Child-Pugh score should be considered for drug dose adjustment according to hepatic clearance. ${ }^{5,6}$ The World Health Organization (WHO) has listed the medications contraindicated in patients with hepatic insufficiency, ${ }^{9}$ but has not defined dose adjustment in patients with impaired liver function. While both the FDA and the EMA demand since 2003 and 2005 respectively, prior to registration performing kinetic studies in patients with cirrhosis if the drug undergoes significant hepatic metabolism, such studies are carried out in patients with preserved liver function (Grade A Child-Pugh) and the results are extrapolated to little dosage adjustments of drugs following a linear dynamics, since in some cases the difference in hepatic metabolism only manifest in grade C Child-Pugh. ${ }^{10}$ Moreover there is controversy about drug dosage or frequency recommended at each level of $\mathrm{CP}^{11}$ since most clinical trials exclude patients with elevated $\mathrm{CP}$ punctuation which corresponds to category $\mathrm{C}$.

The complexity of hepatic metabolism has limited the development of tools allowing predictions of drug behaviour in patients with chronic liver disease. Unlike in renal insufficiency, there are no guidelines and algorithms of reference to facilitate drug dosage in these patients. In this context, there is a growing need to check and expand the available information in the summary of product characteristics. The review of recommendations from other sources and the application of calculation procedures based on the kinetic parameters of the drug are needed to establish and validated practical dose recommendations in chronic liver disease for the frequently used drugs in the care environment. ${ }^{12-15}$

\section{Aim of the study}

The primary objective was to assess the prevalence of prescriptions susceptible to being adjusted in hospitalized patients with liver insufficiency. Secondary objectives were to record pharmaceutical interventions, to evaluate their acceptance and assess the clinical impact on drug efficacy and/or safety.

\section{Ethical approval}

This PI was part of clinical routine and no informed consent or approval from an ethics committee was required. Local rules concerning the patient confidentiality were stated.

\section{Methods}

An uncontrolled prospective evaluation of pharmaceutical intervention was performed in a 920 bed tertiary care university hospital in Spain for a period of 6 months.

\section{Population}

Inclusion criteria: Admission inpatients in the previous 72 hours with analytical parameters of Child-Pugh (ACP) score value $\geq 7$ (Table 1 ), aged $\geq 18$ years, included in the Computerized Prescription Order Entry (CPOE) program (Prescriwin Baxter ${ }^{\circledR} /$ Careview Philips $\left.{ }^{\circledR}\right)$.

Table I Child-Pugh scoring system

\begin{tabular}{llll}
\hline Measure & I point & 2 points & $\mathbf{3}$ points \\
\hline $\begin{array}{l}\text { Total bilirubin } \\
(\mathrm{mg} / \mathrm{dl})\end{array}$ & $<2$ & $2 . \mathrm{I}-3$ & $>3$ \\
$\begin{array}{l}\text { Serum albumin } \\
(\mathrm{g} / \mathrm{dl})\end{array}$ & $>3.5$ & $2.8-3.5$ & $<2.8$ \\
PT INR* & $<I .7$ & I.7I-2.30 & $>2.30$ \\
Ascites & None & Mild & $\begin{array}{l}\text { Moderate to } \\
\text { severe }\end{array}$ \\
$\begin{array}{l}\text { Hepatic } \\
\text { encephalopathy }\end{array}$ & None & $\begin{array}{l}\text { Grade I-II (or } \\
\text { suppressed with } \\
\text { medication) }\end{array}$ & $\begin{array}{l}\text { Grade III-IV (or } \\
\text { refractory) }\end{array}$ \\
\hline
\end{tabular}

*PT INR: prothrombin time international normalized ratio

The ACP (analytical variables only) value was completed by a Pharmacist after reviewing daily the laboratory tests (TDR Bayer ${ }^{\circledR}$ ), written medical records (checking the $\mathrm{CP}$ clinical variables of liver disease: ascites and hepatic encephalopathy) to obtain the final $\mathrm{CP}$ score and determine the appropriate classification category (A, B or C) necessary to make the pharmaceutical interventions due prescribed drugs. 
Exclusion criteria were patients admitted in departments without CPOE (example: emergency service), patients in terminally clinical ill condition, and/or altered analytical results not necessarily related to HI. A review of medical record of patients with ACP $\geq 7$ was performed to confirm that it was not an analytical transient alteration not due to HI [e.g.: factors potentially affecting INR (patients on coumarin derivatives, ribaroxaban, apixaban, vitamin $\mathrm{K}$ deficiency), malnourished (decrease in serum concentrations of proteins and prealbumin and written diagnosis in medical records) or cholestasis (increase in serum concentrations of alkaline phosphatase, gamma glutamyl transpeptidase and written diagnosis in medical records)] without a recorded clinical diagnosis of $\mathrm{HI}$ in medical records.

The score obtained by the ACP can vary from a minimum of 3 to a maximum of 9 points. A cut off $\geq 7$ was taken to identify patients with potential $\mathrm{HI}$ since this ACP score corresponds at least to $\mathrm{CP}$ class B. The CP score employs 5 measures: 3 of them are analytical values indicating liver function [albumin, bilirubin and international normalized ratio (INR) as a derived measure of the prothrombin time] and 2 additional measures are related to disease complications (ascites and encephalopathy). Each variable is scored from 1 to 3 points with 3 indicating the most severe derangement (Table 1) allowing to classify patients in CP classes A, B or C; corresponding to 5-6, 7-9 and 10-15 points respectively).

Pharmaceutical intervention: The PI consisted in a 2 hours daily CPOE review of all the order prescriptions from patients classified $\mathrm{B}$ and $\mathrm{C}$ daily and a dose adjustment recommendation was performed based on a drug database of our own review of hepatic insufficiency. Dosing recommendations were performed according to a literature review (12) on the adjustment of drugs in this population based on a categorization according to their hepatic extraction.

The PI was based on five recommendations: discontinue medication, medication change, dose/frequency change, monitor therapy, or continue the same treatment respectively (there were recorded as an intervention because the patient orders were reviewed for safety reason but no acceptance was required).

The PI was recorded in order to evaluate the efficiency ratio (patients with PI accepted/patients with PI) of the intervention, the acceptance rate and a formal assessment of the clinical impact of NOM. The record of PI was based on established categories depending on the type of PI above and also classified following the NOM (16), defined as health outcomes inappropriate to the objective of pharmacotherapy and/or associated with drug use or therapeutic failure.

The PI clinical impact was evaluated retrospectively by a pharmacist as defined in the Tercer Consenso de Granada regarding NOM classification (16), based on the requirements that all the drugs should fulfil before use: necessity (untreated health problem or effect of unnecessary medicine), effectiveness (non-quantitative or quantitative effectiveness) and safety (non-quantitative or quantitative safety). For example, DRP 6 (the patient suffers from a health problem as a consequence of a quantitative safety problem of the medication) is related with a NOM quantitative safety problem (the patient suffers from a health problem associated with a quantitative safety problem of the medication).

The physicians were informed via a text message in the CPOE program or, if necessary, they were contacted personally by phone or in person. Acceptance of the interventions was measured based on modifications made in the CPOE at 72 hours and was classified as accepted or not, except the PI defined as "same treatment".

Statistical analysis: Mean and standard deviation for quantitative and relative frequencies for quantitative variables were used. The study data were collected and statistically performed in Excel $\circledR^{\circledR}$.

\section{Results}

During the study period of 6 months (September 2011-February 2012), 8,815 patients were recorded, 171 of them had an ACP value $\geq 7$ (corresponding to 353 blood test records).

On applying the exclusion criteria 141 patients were ruled out for the following reasons: treatment with potentially affecting INR drugs (46 patients), malnourished (23), cholestasis criteria (17), and clinical services without CPOE (47), and for critical condition (8), which was determinate by the clinical physician and recorded on patient chart.

Finally, our potential interventional population was 30 patients (57.5 years (21-82), 25 men (83.3\%) and mean hospital stage $9.7 \pm 10.9$ days) with 535 orders to review. The main causes of HI in these patients were: 12 chronic $\mathrm{C}$ hepatitis virus infection, $9 \mathrm{HI}$ due alcohol, 5 drug related liver toxicity and $4 \mathrm{~B}$ and $\mathrm{C}$ chronic hepatitis virus coinfection. The mean blood test parameter distribution was: bilirubin $5.5 \mathrm{mg} / \mathrm{dL}+5.4$, albumin $25.9 \pm 4.5 \mathrm{~g} / \mathrm{L}$ and INR $1.6 \pm 0.4$. Once clinical variables (ascites and encephalopathy) and the clinical history had been reviewed the final CP classification was: B (11 patients) and C (19 patients)

The 30 patients were admitted to the following Departments: Gastroenterology (21); Internal Medicine (4); Cardiology (2); Nephrology (1) Haematology (1) and General Surgery (1). The actual prevalence of liver disease during the study period (determined by the International Classification Disease (ICD-9) diagnostic criteria) was 386 cases of total hospital admissions $(4.4 \%)$. The HI prevalence rate obtained by the CP screening in our study was $0.34 \%$ of the recorded hospital admissions.

In 30 assessed patients (mean of $17.8+2.7$ prescription orders per admission), 535 prescription orders corresponding to 127 drugs were performed. Three hundred and eighteen orders $(59.4 \%$ of total), corresponding to a total of 49 different drugs ( $38.6 \%$ of total), according to the recommendations based on our own review of HI drug database (12), were candidates for a PI (Figure1): requiring dose adjustment 67 orders ( 8 drugs), use with caution 43 orders (9 drugs), contraindicated 29 orders (9 drugs), analytical or pharmacological monitoring 47 orders ( 3 drugs) and to continue the same treatment (no need dose adjustment for the individual patient CP score ) 132 orders (20 drugs).

One hundred and eighty-six PI were made on 30 patients $(6.2$ $\mathrm{PI} /$ patient), corresponding to anatomical therapeutic chemical classification system and drugs involved shown in Table 2. The PIs performed were classified as: dosage/frequency change $67 \mathrm{PI}$, pharmacological/analytical monitor 47, caution 43 and contraindications 29 . On average we recorded a 0.97 contraindicated order/patient. The main drugs to be adjusted or avoided according to the recommendations based on a drug database review of HI (12) were morphine (12), fluconazole (6), acetylsalicylic acid (4) and a miscellanea of drugs (7). The classification and justifying reason for PI can be seen detailed in Table 3. Acceptance level was finally made in 143 PI (Caution was included as a PI but not valuable acceptance was done): 99 PI were accepted (99/143: 69.7\%) and 44 not accepted (44/143: $30.3 \%)$. 
Table 2 Potential pharmaceutical intervention classified by the anatomic and therapeutic chemical drug classification system and the drugs involved

\begin{tabular}{llll}
\hline $\begin{array}{l}\text { Anatomical therapeutic chemical } \\
\text { (ATC) classification system }\end{array}$ & Drugs & $\begin{array}{l}\mathbf{P I} \\
(\mathbf{n = 1 8 6 )}\end{array}$ & \% \\
\hline Antiinfectives for systemic use & $\begin{array}{l}\text { Abacavir/lamivudine, abacavir, amoxicillin-clavulanic, } \\
\text { caspofungin, cloxacillin, efavirenz, emtricitabine/tenofovir/ } \\
\text { efavirenz, fluconazole, fosamprenavir, levofloxacin, } \\
\text { metronidazol, raltegravir, rifampicin, ritonavir, saquinavir, } \\
\text { sulfamethoxazole/trimethoprim }\end{array}$ & 40 & 21.5 \\
Nervous system & $\begin{array}{l}\text { Codeine, haloperidol, morphine, acetaminophen } \\
\text { acetylsalicylic acid, enalapril, furosemide, }\end{array}$ & 37 & 30.6 \\
hydrochlorothiazide & 50 & 26.88 \\
Alimentary tract and metabolism & Metformin, propranolol, ranitidine & 33 & 17.7 \\
$\begin{array}{l}\text { Antineoplastic and immunomodulating } \\
\text { agents }\end{array}$ & Ciclosporin & 3 & 1.6 \\
Musculoskeletal system & Allopurinol & 3 & 1.6 \\
\hline
\end{tabular}

Table 3 Pharmaceutical intervention classification and clinical impact

\begin{tabular}{lll}
\hline Pharmaceutical intervention & Clinical impact & $\mathbf{n}$ \\
\hline I. Discontinue drug & & 23 \\
I.I. Contraindication & Safety & 4 \\
I.2. Adverse reaction prevention & Safety & \\
2. Drug change & & 2 \\
2.1. Contraindication & Safety & \\
3. Dose, frequency change & & 67 \\
3.1. Overdose & Safety & \\
4. Monitoring & & 47 \\
4.I. Blood test monitoring & Effectiveness/safety & 43 \\
5. Caution & Safety & \\
\hline
\end{tabular}

Results are expressed in number of interventions.

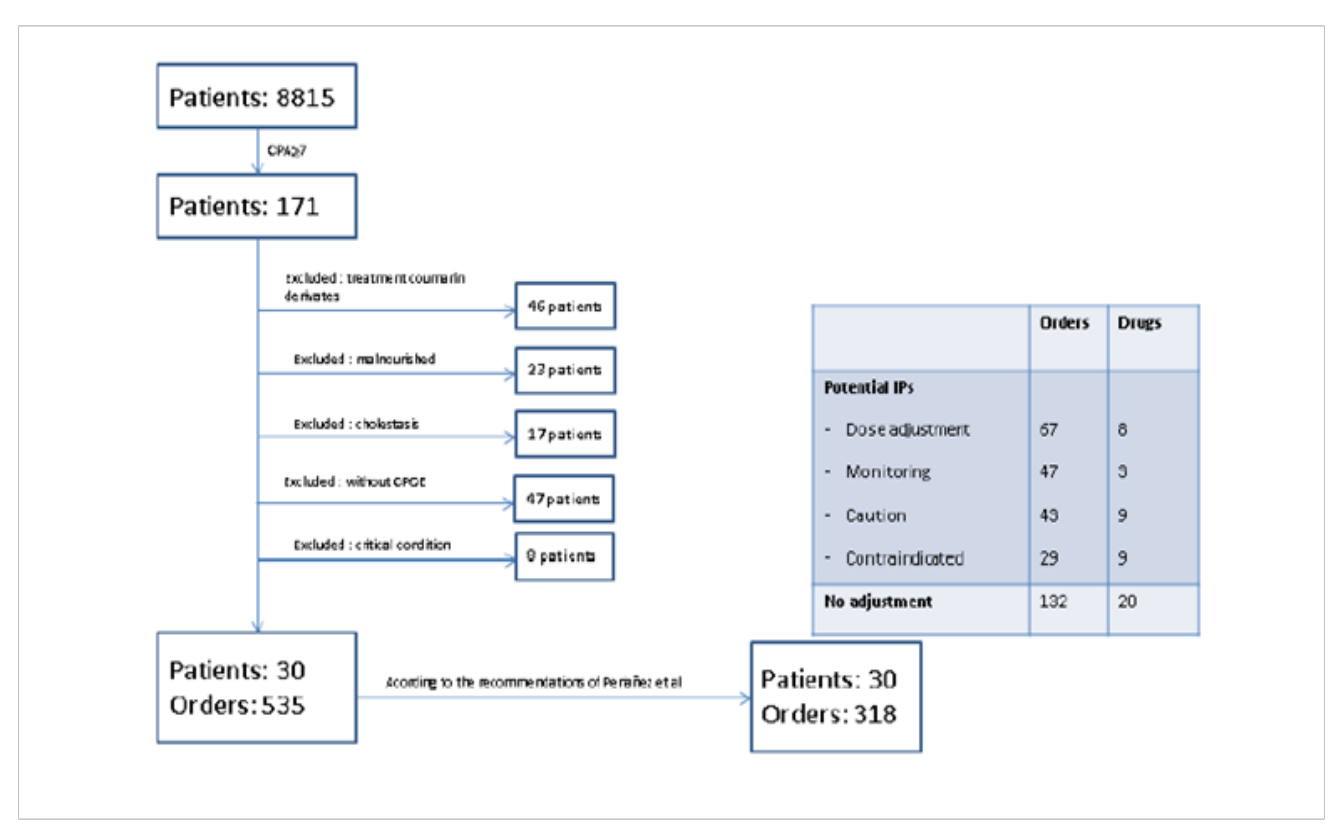

Figure I Pharmaceutical interventions in flow chart.

Citation: Párraga LP, Bosch PV, Martínez-López I, et al. Evaluation of pharmaceutical intervention in patients with hepatic insufficiency in a hospital setting. Gastroenterol Hepatol Open Access. 2018;9(6):24I-247. DOI: 10.15406/ghoa.20 18.09.00335 
The dosage/frequency change PI was accepted in 54/67(81.8\%) of cases, drug discontinuation in $22 / 29(75.1 \%)$ and the pharmacological/ analytical monitor in 23/47 (48.9\%). The PI acceptance, classified by anatomical therapeutical chemical (ATC) classification system groups, was: Antiinfectives for systemic use: 39 (39.4\%), Nervous system: 36 (36.4\%), Cardiovascular system: 17 (17.2\%), Alimentary tract and metabolism: 3 (3.0\%), Miscellanea: 2 (2.0\%) and Musculoskeletal system: $2(2.0 \%)$.

The highest number of recorded acceptances by clinical Departments was: Gastroenterology (51), followed by Internal Medicine (25) and Intensive Care Department (21). The efficiency ratio was $73.3 \%(22 / 30)$. Regarding the intervention mode, 116 were made as a written message at the CPOE electronic prescribing program and 27 were personally contacted.

Regarding the Tercer Consenso de Granada ${ }^{16}$ the PI performed had a potential impact on NOM drug effectiveness of $12.6 \%$ [nonquantitative (66.7\%) and quantitative (33.3\%)] and safety on $87.4 \%$ [non-quantitative $(46.5 \%)$ and quantitative $(53.5 \%)$ ]. No PI had impact on necessity (untreated health problem or effect of unnecessary medicine).

\section{Discussion}

The study reported important exploratory findings due to inappropriate prescriptions in patients with liver disease involvement in medication safety. To our knowledge, this is the first prospective interventional study reporting the PI potential effect based on drug dose adjustments in patients with hepatic insufficiency.

Although since 1997, the CP classification has been used to establish criteria for liver transplantation ${ }^{17}$ and to estimate its impact on pharmacokinetics and in drug dosing recommendations,${ }^{18}$ it is not used systematically in the detection of HI patients. Alternatively the model for end-stage liver disease (MELD) score is used to predict survival of patients with liver cirrhosis and to determine the urgency of liver transplantation. ${ }^{19,20}$ Despite MELD score includes reproductive variables (serum creatinine, bilirubin, INR) avoiding distortion caused by ambiguous clinical assessment, it has not been extensively evaluated whether the MELD score will predict individual elimination capacity in patients with liver cirrhosis and might thus help guiding drug dose adjustments.

Franz et al. ${ }^{21}$ published a similar but retrospective study based on the prevalence of DRP. About $20 \%$ of orders were considered inaccurate or contraindicated, showing a higher frequency of DRP related with hospitalization. Similar pharmacological therapeutic classes and drugs were mostly involved in both studies in patients with cirrhosis. Nevertheless, there are important methodological differences with our study. In the former study only cirrhotic patients were included, while in our case we considered different patients with impaired liver disease, (cirrhotic patients, alcoholic, acute/chronic hepatitis, liver failure,...) and diverse liver fibrosis like other published studies. ${ }^{22,23}$

The efficiency ratio of our study was higher $(73.3 \%)$ than in other studies describing values from $10-29 \% .^{24-26}$ However there are differences both in the context of the research setting (different clinical departments) and the different methodology employed. ${ }^{7}$ The PI acceptance rate was $69.7 \%$, similar to other studies, ranging from $64 \%-75 \% .^{26-30}$ The evaluation of the acceptance rate was based mainly on the modifications done in the CPOE, but unfortunately there was not a control group or a feedback from the clinician, except when they were contacted personally. The clinicians were mainly notified in the afternoon, through a written message, which probably influenced the acceptance rate; firstly because the clinician could not read the message in the CPOE program, and secondly because previous experience shows that the greatest acceptance rates are obtained by direct verbal communication with the physician. . $7,28,31,32^{2}$

The benefit/risk balance made by the clinician, and the absence of therapeutic alternatives may justify the rejection of the PI. There was also a high number of potential PI where we decided not to intervene, mainly because of the terminally ill condition of patients.

Our study shows that $58.5 \%$ of orders were considered for potential PI regarding the impaired liver function, associated with a higher probability of DRP. Therefore it seems that clinicians were aware of high-risk medications in these patients and the need for dose adjustment in patients with hepatic insufficiency. One contributing factor could be that the maintenance dose of such drugs can be adjusted according to the clinical effect and tolerance of each individual patient, and thus leading to closer monitoring in this population.

Regarding the clinical potential impact of the PI, regarding NOM classification, ${ }^{16}$ based on the Tercer Consenso de Granada $12.6 \%$ potentially influenced the inefficacy of treatment and $87.4 \%$ the safety. Both percentages are different to those described in other studies ${ }^{24,25,33}$ reporting effectiveness and safety values ranging from $48-58 \%$ and $38-49 \%$ respectively. In the study by Fernandez et al., ${ }^{34}$ those drugs related NOM, avoided by performing PI had a higher impact on patient safety, as we found in our study. Other recently published data,${ }^{32}$ show a similar distribution between the percentages of efficacy and safety.

Interestingly, in the study of Franz et al. ${ }^{21}$ they found a similar drug class profile to the data found in our study. Indeed, DRPs associated with inadequate drug dose were more related to pharmacodynamic impairment in patients with liver cirrhosis, and mainly included the following classes: NSAIDs, sedatives/hypnotics and oral antidiabetics. Also, drugs acting on the central nervous system had a higher risk of causing hepatic encephalopathy, probably decompensating the underlying disease and consequently needing a particular attention.

\section{Strengths and limitations}

In our study we proceeded to use blood tests data to pre-screen patients. This detection system under-reported patients with chronic liver disease. Indeed, some patients cannot have impaired analytical blood tests, and however present HI. Moreover, patients without HI may have altered analytical blood tests so it was necessary to perform the clinical follow-up of patients with ACP $\geq 7$ to confirm that it was not an analytical transient alteration due to HI. The absence of a control group could jeopardize acceptance of IP for standard practice. The clinicians were mainly notified in the afternoon, through a written message, which probably influenced the acceptance rate.

\section{Implications for practice}

Based on the evaluation, we have detected that there is a lack on drug safety in patients with HI. Clinical pharmacists are medication experts with direct contact with patients and thus potentially have an important role to play in closing drug safety.

More research is needed to understand about the safety of drugs in patients with decompensated HI with a systematic and standardized database linked to an automatic tool. In addition, the ease of obtaining 
a score to perform an effective, validated and reproducible prescreening to established patients with liver disease would help focus efforts. As a follow up to this study, we are planning to conduct an in depth qualitative study to address these issues.

\section{Conclusion}

It is necessary to implement a systematic population intervention program to stratify patients according to their HI class, allowing us to recommend an appropriate dose in $\mathrm{HI}$ and avoid ordering hepatotoxic drugs. The results of our study show that about one third of the prescriptions in patients with $\mathrm{HI}$ are candidates for PI, mostly due to contraindications or wrong dosage. As the pharmacists' intervention is widely accepted and appreciated by the medical community they have an important role to play by increasing the safe use of drugs.

\section{Acknowledgements}

None.

\section{Conflict of interest}

The author declares there is no conflict of interest.

\section{Funding}

This study had no specific source of funding.

\section{References}

1. Westphal JF, Brogard JM. Drug administration in chronic liver disease. Drug Saf. 1997;17(1):47-73.

2. Bennett WM, Singer I, Coggins CJ. A guide to drug therapy in renal failure. JAMA. 1974;230(11):1544-1553

3. Chertow GM, Lee J, Kuperman GJ, et al. Guided medication dosing for inpatients with renal insufficiency. JAMA. 2001;286(22):2839-2844.

4. Hug BL, Witkowski DJ, Sox CM, et al. Occurrence of adverse, often preventable, events in community hospitals involving nephrotoxic drugs or those excreted by the kidney. Kidney Int. 2009;76(11):1192-1198.

5. EMEA. Guideline on the evaluation of the pharmacokinetics of medicinal products in patients with impaired hepatic function. 2005.

6. FDA. Guidance for industry: pharmacokinetics in patients with impaired hepatic function: study design, data analysis, and impact on dosing and labelling. 2003.

7. Sistanizad M, Peterson GM. Use of contraindicated drugs in patients with chronic liver disease: a therapeutic dilemma. Int J Clin Pharmacol Ther. 2013;51(1):1-4.

8. Pugh RN, Murray-Lyon IM, Dawson JL, et al. Transection of the oesophagus for bleeding oesophageal varices. Br J Surg. 1973;60(8):646649.

9. World Health Organization. WHO Model Formulary 2008. Stuart MC, Kouimtzi M, Suzanne R. Hill D of EM and P, Policies, World Health Organization G, editors. World Health Organization; 2008. 664 p.

10. Brockmöller J, Thomsen T, Wittstock M, et al. Pharmacokinetics of levetiracetam in patients with moderate to severe liver cirrhosis (ChildPugh classes A, B, and C): Characterization by dynamic liver function tests. Clin Pharmacol Ther. 2005;77(6):529-541.

11. Spray JW, Willett K, Chase D, et al. Dosage adjustment for hepatic dysfunction based on Child-Pugh scores. Am J Health Syst Pharm. 2007;64(7):690, 692-693.
12. Periáñez-Párraga L, Martínez-López I, Ventayol-Bosch P, et al. Drug dosage recommendations in patients with chronic liver disease. Rev Esp Enferm Dig. 2012;104(4):165-184.

13. Delcò F, Tchambaz L, Schlienger R, et al. Dose adjustment in patients with liver disease. Drug Saf. 2005;28(6):529-545.

14. Huet PM, Villeneuve JP. Determinants of drug disposition in patients with cirrhosis. Hepatology. 1983;3(6):913-918.

15. Krähenbühl S, Reichen J. Pharmacokinetics and Pharmacodynamics in Cirrhosis. Medicine (Baltimore). 2002;30(11):24-27.

16. Third Consensus of Granada on Drug Related Problems (DRP) and NegativeOutcomes associated with Medication (NOM). Ars Pharmaceutica. 2007. p. 5-17.

17. Reuben A. Child comes of age. Hepatology. 2002;35(1):244-245.

18. Forman LM, Lucey MR. Predicting the prognosis of chronic liver disease: an evolution from child to MELD. Mayo End-stage Liver Disease. Hepatology. 2001;33(2):473-475.

19. Wiesner RH, McDiarmid S V, Kamath PS, et al. MELD and PELD: application of survival models to liver allocation. Liver Transpl. 2001;7(7):567-580.

20. Kamath PS, Kim WR. The model for end-stage liver disease (MELD). Hepatology. 2007;45(3):797-805.

21. Franz CC, Hildbrand C, Born C, et al. Dose adjustment in patients with liver cirrhosis: impact on adverse drug reactions and hospitalizations. Eur J Clin Pharmacol. 2013;69(8):1565-1573.

22. Dominguez S, Ghosn J, Peytavin G, et al. Impact of hepatitis C and liver fibrosis on antiretroviral plasma drug concentrations in HIV-HCV co-infected patients: the HEPADOSE study. J Antimicrob Chemother. 201;65(11):2445-2449.

23. Meynard J-L, Lacombe K, Poirier J-M, et al. Influence of liver fibrosis stage on plasma levels of efavirenz in HIV-infected patients with chronic hepatitis B or C. J Antimicrob Chemother. 2009;63(3):579-584.

24. Gutiérrez Paúls L, González Alvarez I, Requena Caturla T, et al. Prescription errors in patients admitted to an internal medicine department from the emergency room. Farm Hosp. 2015;30(2):85-91.

25. Izco N, Codina $C$, Tuset $M$, et al. Evaluation of the integration of the pharmacist in care teams of hospitalization units. Farm Hosp. 2002;26:18-27.

26. Gorgas Torner MQ, Odena Estradé E, Pastor Solernou F. Pharmaceutical care for drug-related problems in inpatients. Farm Hosp. 2003;27(5):280289.

27. Fernández-Díaz E, Goikolea-Ugarte FJ, Domingo-Echaburu S, et al. Implantation of a posology adjustment program in patients with decreased renal function. Pharmaceutical Care. 2007. p. 102-106.

28. McMullin ST, Reichley RM, Kahn MG, et al. Automated system for identifying potential dosage problems at a large university hospital. Am J Health Syst Pharm. 1997;54(5):545-549.

29. Goldberg DE, Baardsgaard G, Johnson MT, et al. Computer-based program for identifying medication orders requiring dosage modification based on renal function. Am J Hosp Pharm. 1991;48(9):1965-1969.

30. Campos Vieira N, Bicas Rocha K, Calleja Hernández MA, et al. Seguimiento farmacoterapéutico en pacientes ingresados en el Servicio de Medicina Interna del Hospital Infanta Margarita. Farm Hosp. 2004;28(4):251-257.

31. Falconnier AD, Haefeli WE, Schoenenberger RA, et al. Drug dosage in patients with renal failure optimized by immediate concurrent feedback. J Gen Intern Med. 2001;16(6):369-375. 
32. Gastelurrutia P, Benrimoj SI, Espejo J, et al. Negative clinical outcomes associated with drug-related problems in heart failure (HF) outpatients impact of a pharmacist in a multidisciplinary HF clinic. J Card Fail. 2011;17(3):217-223.
33. Clopés A CI. Pharmaceutical Interventions (Part II): Validation of the methodology used to measure the impact. Farm Hosp. 2000;24:215-220.

34. Fernandez-Llimos F, Faus MJ. From "drug-related problems" to "negative clinical outcomes". Am J Health Syst Pharm. 2005;62(22):2348, 2350. 